\title{
Immunocytochemical Localization of Arachidonate 15-Lipoxygenase in Erythrocytes, Leukocytes, and Airway Cells
}

\author{
J. A. Nadel, ** D. J. Conrad, I. F. Ueki, A. Schuster, and E. Sigal* \\ Cardiovascular Research Institute and Departments of ${ }^{*}$ Medicine and ${ }^{\ddagger}$ Physiology, \\ University of California, San Francisco, San Francisco, California 94143
}

\begin{abstract}
In reticulocytes, the enzyme 15-lipoxygenase (15-LO) is believed to contribute to cellular differentiation, and in leukocytes and airway cells 15-LO generates inflammatory mediators. The recent availability of antibodies to 15-LO now allows us to determine which specific cells contain the enzyme, to characterize its subcellular localization, and to determine its expression at the translational level. A polyclonal antibody to recombinant human reticulocyte 15-LO was used with a standard immunofluorescent technique. In rabbit red blood cells, fluorescence appeared during the course of anemia. Early reticulocytes did not fluoresce, but more mature reticulocytes showed increased fluorescent intensity. Late reticulocytes contained little fluorescence. Among human leukocytes, only eosinophils fluoresced. In human trachea, 15-LO immunofluorescence was localized to epithelial cells, and both basal and ciliated cells fluoresced. In all cells studied, fluorescence was localized to the cytoplasm and was variable in degree among cells in each preparation. We conclude that the 15-LO of airway cells and eosinophils is immunologically related to the reticulocyte 15-LO. Furthermore, the variable fluorescence among cells (e.g., in epithelium) and during development (e.g., reticulocytes) suggests a role of 15LO in cell growth and development. (J. Clin. Invest. 1991. 87:1139-1145.) Key words: reticulocyte lipoxygenase $\bullet$ arachidonic acid • airway epithelium • eosinophil
\end{abstract}

\section{Introduction}

The enzyme 15 -lipoxygenase $(15-\mathrm{LO})^{1}$ catalyzes the insertion of molecular oxygen into arachidonic acid at carbon 15 . The enzyme can also oxygenate other polyenoic free fatty acids as well as a variety of phospholipids (1). This ability to perform lipid peroxidation is evident in multiple biological systems. For example, 15-LO activity has been identified by enzymatic assays in rabbit and human reticulocytes $(2,3)$, in human leukocytes (4-6), in human lung (7), and in human airway epithelial cells (8). In the reticulocyte, the enzyme is believed to contribute to differentiation of that cell into a mature erythrocyte. The mechanism of action is presumably the hydroperoxidation of

Address correspondence to Jay A. Nadel, M.D., Cardiovascular Research Institute, Box 0130, University of California, San Francisco, CA 94143-0130.

Received for publication 10 August 1990 and in revised form 16 November 1990

1. Abbreviations used in this paper: HETE, hydroxylcosatetraenoic acid; LO, lipoxygenase; OS, osteosarcoma.

J. Clin. Invest.

(c) The American Society for Clinical Investigation, Inc.

0021-9738/91/04/1139/07 \$2.00

Volume 87, April 1991, 1139-1145 intracellular membranes that are then further degraded via proteolysis $(2,9)$. In airway cells and in leukocytes, 15-LO is believed to act via the metabolism of arachidonic acid, thereby generating potent inflammatory mediators which have effects on various tissues in the airways (10-14).

These potential biological actions of 15-LO have led to an increasing interest in understanding the biochemical mechanism of the enzyme. The mammalian form of the enzyme has been purified to homogeneity from rabbit reticulocytes (2) and from human leukocytes (15). Using protein sequence from the mammalian enzymes, we recently isolated a cDNA encoding 15-LO from a human reticulocyte library (16). This cDNA has now been expressed in active form in both eukaryotic and prokaryotic cells, verifying its authenticity (17).

The precise localization of 15-LO in blood cells and in lung has not yet been defined. Prior studies using enzymatic activity have failed to accomplish this because they have been limited in their ability to detect the expression of the enzyme on a cellular level. Tissue localization of $15-\mathrm{LO}$ has the potential to yield important information regarding the expression of the enzyme and regarding additional biological roles of the enzyme within a given cell type or tissue. Bacterial expression of recombinant 15-LO has allowed us to obtain sufficient quantities of recombinant enzyme to develop a polyclonal antibody to human reticulocyte 15-LO (17) and to study its immunocytochemical localization in human leukocytes and airways and in rabbit red blood cells.

\section{Methods}

Preparation of cells. Human osteosarcoma (OS) cells were transfected with a mammalian expression vector containing the cDNA for human reticulocyte $15-\mathrm{LO}$ as previously described (17). This cell line, OS15LOX (+), has been shown to express $15-\mathrm{LO}$ by enzymatic activity and by immunoreactivity (17). The cell line OS15LOX $(-)$ was created by transfecting osteosarcoma cells with the same mammalian expression vector but without the 15-LO cDNA and was used in this study to assess baseline immunofluorescence in osteosarcoma cells.

In preliminary studies, we found that normal human red blood cells did not show 15-LO immunofluorescence. Similarly, blood from nonanemic rabbits showed no 15-LO immunofluorescence. Therefore, we bled three rabbits intermittently $(30-50 \mathrm{ml} /$ bleed) for up to $15 \mathrm{~d}$. After day 7 of bleeding, the anemia was stable (hematocrit, 20-25\%; reticulocyte count, $>25 \%$ ). In the first rabbit, we studied the peripheral blood on day 6 of bleeding, and we compared 15-LO fluorescence to that obtained in red blood cells removed on day 1 . In the second rabbit, we characterized the time course of 15-LO fluorescence in relation to the evolution of the anemia. In the third rabbit, specimens of venous blood were removed on days 10 and 15 of bleeding, and samples of blood were separated into four fractions by a method previously described (18), which separates red blood cells according to their state of maturation. In these four fractions, we studied cell morphology and 15-LO fluorescence. All blood smears were fixed with methanol or cold acetone for immunofluorescence. 
Human leukocytes were obtained from anticoagulated venous blood samples from two normal volunteers and from four hypereosinophilic patients. Leukocytes were separated by dextran sedimentation, followed by hypotonic lysis of the red blood cells. Cytospin preparations of the buffy coat were fixed in methanol. Various leukocyte types were identified by phase microscopy of blood smears and then were compared for the presence of 15-LO by fluorescent microscopy. Neutrophils and eosinophils were further separated from mononuclear cells by Ficoll-Paque or Percoll gradients (19) and were analyzed for both $15-\mathrm{LO}$ activity and immunofluorescence. Differential cell counts on 200 cells were obtained from blood smears after Diff-Quik staining (Dade Diagnostics, Aguada, Puerto Rico).

Human airway epithelial cells were examined in three ways. First, we obtained airway epithelial cells from scrapings of the mucosa from two tracheas postmortem. Second, human airway epithelial cells ( $>95 \%$ purity) were isolated from a human trachea obtained $<24 \mathrm{~h}$ postmortem by a method of enzymatic digestion (20). Aliquots of all isolated cells were cytocentrifuged and fixed for immunofluorescence with methanol. Third, we obtained bronchial tissue from one patient at the time of lung resection and tracheal tissue from two patients postmortem. Human airway tissue obtained from tissue resections from operations and postmortem tissue were embedded in OCT (TissueTek; Miles Inc., Diagnostic Division, Elkhart, IN) and frozen in Freon and liquid nitrogen. The tissues, $8-10 \mu \mathrm{m}$ thick, were cut on a cryostat (IEC Minotome; International Equipment Co., Needham Heights, MA), placed on chrome-alum gelatin-coated slides, and stored at $-80^{\circ} \mathrm{C}$ until immunocytochemistry was performed.

Antibody to human 15-lipoxygenase. A previously characterized rabbit polyclonal antibody to recombinant human reticulocyte 15-LO was used in this study (17). In brief, the cDNA for 15-LO was subcloned into a bacterial expression vector and was expressed as a fusion with the CheY protein. The overexpressed protein was purified from bacteria and injected into rabbits. This recombinant protein was also used to test whether positive immunofluorescence could be inhibited selectively. The antibody to this recombinant material was purified by affinity chromatography (Sepharose-G, Pharmacia Fine Chemicals, Piscataway, NJ) and was shown to specifically react on immunoblots to one major band of the expected molecular size $(70,000 \mathrm{D})$ for $15-\mathrm{LO}$ from human leukocytes, human tracheal epithelial cells, and rabbit reticulocytes (17). It did not cross-react with purified 5-LO (17) or to highly enriched preparations of platelet 12-LO (Sigal, E., unpublished observations).

Immunofluorescence staining. Immunocytochemistry was performed at room temperature in a humidified chamber. Cells and tissues were first rinsed with PBS and were incubated with an excess of BSA or host serum for $\mathbf{3 0}$ min to inhibit nonspecific staining. Appropriate dilutions of the polyclonal 15-LO antibody were incubated on the tissue sections for $1 \mathrm{~h}$ and then rinsed extensively with PBS to eliminate unbound antibody. The sections were then exposed for $\mathbf{3 0}$ min to a second antibody conjugated with fluorescein isothiocyanate and were again washed to remove the unbound fluorescent tracer. Sections were coated with the fluorescent enhancer DABco and were viewed under a Zeiss Axioplan fluorescent microscope. For the study of eosinophils, an additional step was included to prevent nonspecific staining, as described elsewhere (21): After exposure to the primary antibody, eosinophils were further incubated with a $1 \%$ chromotrope $2 \mathrm{R}$ solution at $37^{\circ} \mathrm{C}$ for $30 \mathrm{~min}$. After extensive washes, the tissue sections were treated in the same fashion as above.

Assay of 15-lipoxygenase activity. Cellular capacity for oxygenation of exogenous substrate was tested by incubating cells with arachidonic acid dissolved in ethanol $(0.5 \%)$. Final concentrations of $\sim 5 \times 10^{6}$ cells $/ \mathrm{ml}$ were incubated with substrate $(160 \mu \mathrm{M})$ for $15 \mathrm{~min}$ at $37^{\circ} \mathrm{C}$. In some experiments, broken cell preparations were prepared by sonication as described elsewhere (15). Cell suspensions were extracted with 1 vol of 2-propanol containing 1.2\% acetic acid (vol/vol) (pH 3.5) and 1 vol of chloroform. An aliquot of prostaglandin $B_{2}$ was used as an internal standard. All extracts were washed with water and were reconstituted in methanol for storage at $-70^{\circ} \mathrm{C}$ under $\mathrm{N}_{2}$. Reagents were incu- bated with cell-free media to assess nonenzymatic generation of products. Extracts were reconstituted in chromatography solvent and were analyzed by reverse-phase (RP) HPLC on a model 840 chromatography system (Waters Chromatography Division, Milford, MA) using a Dynamax C-18 column $(5 \mu \mathrm{m}, 4.6 \mathrm{~mm} \times 25 \mathrm{~cm}$, Rainin, Instrument Co., Inc., Emeryville, CA) as previously described (17).

\section{Results}

We established conditions for immunofluorescent staining of mammalian tissues by studying the human osteosarcoma cell line, OS15LOX(t). Transfection of these cells with a mammalian expression plasmid containing the cDNA for human reticulocyte 15-LO produced cells capable of oxidizing arachidonic acid into 15-LO products. Immunofluorescent examination of these cells showed no fluorescence in the presence of an equal dilution of nonimmune $\mathrm{IgG}$, but the $\mathrm{OS} 15 \mathrm{LOX}(+)$ cells showed positive fluorescence when they were exposed to the 15-LO antibody. The fluorescence was localized to the cytoplasm, with no fluorescence in the nuclear region. The fluorescence varied markedly among cells in the preparation. Quenching of fluorescence occurred in a concentration-dependent fashion by an excess of recombinant human 15-LO. In contrast, no significant fluorescence was obtained in a companion osteosarcoma cell line, OS15LOX(-), which was transfected with the same expression vector but which did not contain the 15-LO cDNA and did not express 15-LO activity.

Rabbit red blood cells. Mature human and rabbit red blood cells (hematocrits $>30 \%$, reticulocyte counts $<2 \%$ ) did not show immunofluorescence (data not shown). However, in three rabbits, after repeated bleeding (30-50 ml/bleed for up to $2 \mathrm{wk}$ ), the animals developed a significant anemia which became stable after day 7 of bleeding (hematocrit, $\leq 25 \%$; reticulocyte count, $>25 \%$ ). The studies differed in the three rabbits. In the first rabbit, we compared blood removed on day 6 with blood removed on day 1 of bleeding. On day 1, no 15-LO immunofluorescence was evident on the blood smears. However, smears from day 6 showed many red blood cells that exhibited strongly positive 15-LO fluorescence. The degree of fluorescence varied widely among cells. The fluorescent cells were identified as reticulocytes when stained with new methylene blue. Both purified rabbit 15-LO and human recombinant 15-LO inhibited the fluorescence in a concentration-dependent fashion. Nonimmune IgG in equivalent dilutions produced no fluorescence.

In the second rabbit, we obtained sequential blood samples over the 2-wk period of bleeding. Although the reticulocyte count had increased early in the course of bleeding, positive immunofluorescence lagged. Thus, on day 3 of bleeding, no positive fluorescence was found (Fig. 1, left). The number of reticulocytes with positive fluorescence increased progressively to day 8 (Fig. 1, middle) and then gradually decreased. Thus, although the number of reticulocytes remained constant from day 8 to 13 , both the number of reticulocytes that fluoresced and the degree of fluorescence had decreased by day 13 (Fig. 1, right). These studies indicate that $15-\mathrm{LO}$ expression changes during the differentiation of reticulocytes.

In the third rabbit, we obtained blood samples on days 10 and 15 , and we separated the cells by buoyant density, a method previously described for separating red blood cells according to their state of maturation (Fig. 2) (18). The first fraction, containing early reticulocytes, showed little fluorescence. 

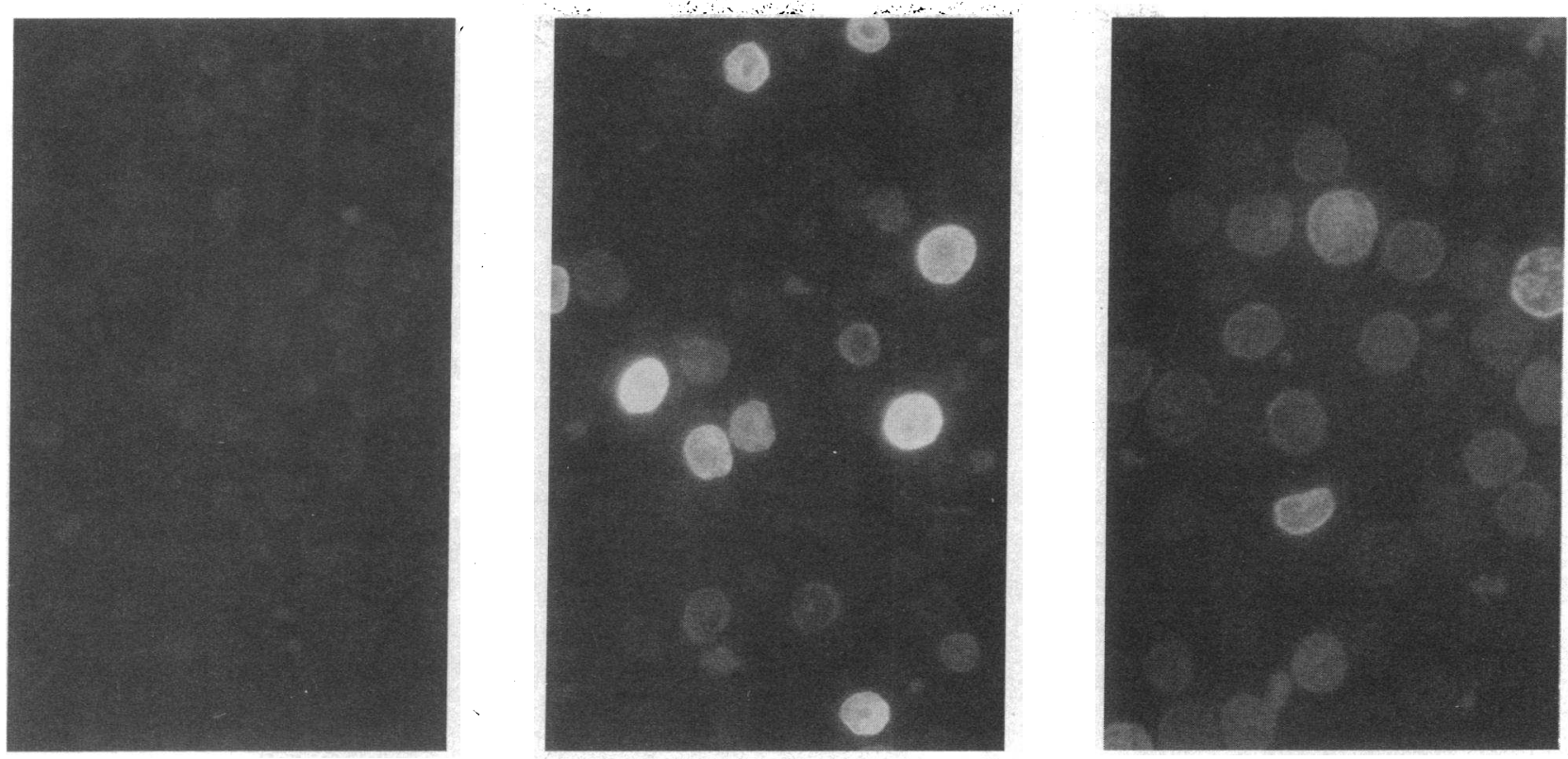

Figure 1. Photomicrographs of 15-lipoxygenase immunofluorescence in peripheral blood removed from a rabbit in day 3 (left), day 8 (middle), and day 13 (right) during repeated bleeding.

Fractions 2 and 3 contained reticulocytes of intermediate size (and age), and many of these reticulocytes showed intense 15LO fluorescence. The fourth fraction contained the smallest reticulocytes, which fluoresced only slightly. Some of these cells showed a rim of concentrated fluorescence at the periphery of the cell, perhaps due to their biconcave disc shape. These studies indicate that early. reticulocytes do not contain 15-LO, that its expression increases over several days of maturation, and that in late reticulocytes the expression of $15-\mathrm{LO}$ is again decreased.

Human eosinophils. It is clear that eosinophils have an active 15-LO (4), but it is not clear whether other granulocytes such as neutrophils express 15-LO. Therefore, in two normal and in four hypereosinophilic patients, we obtained blood, concentrated the leukocytes, prepared blood smears, and examined them microscopically. Monocytes, lymphocytes, and neu- trophils were identifiable on phase microscopy (Fig. 3, left) and did not exhibit 15-LO immunofluorescence (Fig. 3, right). Eosinophils were also identifiable by the typical morphology of the nucleus and the large refractile granules (Fig. 3, left); only eosinophils exhibited 15-LO immunofluorescence (Fig. 3, right).

In each of the six subjects, we further isolated neutrophils and eosinophils from other leukocytes on gradients, prepared smears of the cells, and examined them for percent neutrophils vs. eosinophils and for 15-LO immunofluorescence. Separate aliquots of cells were incubated with arachidonic acid, and 15LO activity was subsequently assessed (Table I). In the two specimens containing $<1 \%$ eosinophils, no 15 -LO products were detected. Only blood containing eosinophils was capable of generating 15-LO products. When the values of these products were normalized for the numbers of eosinophils present,
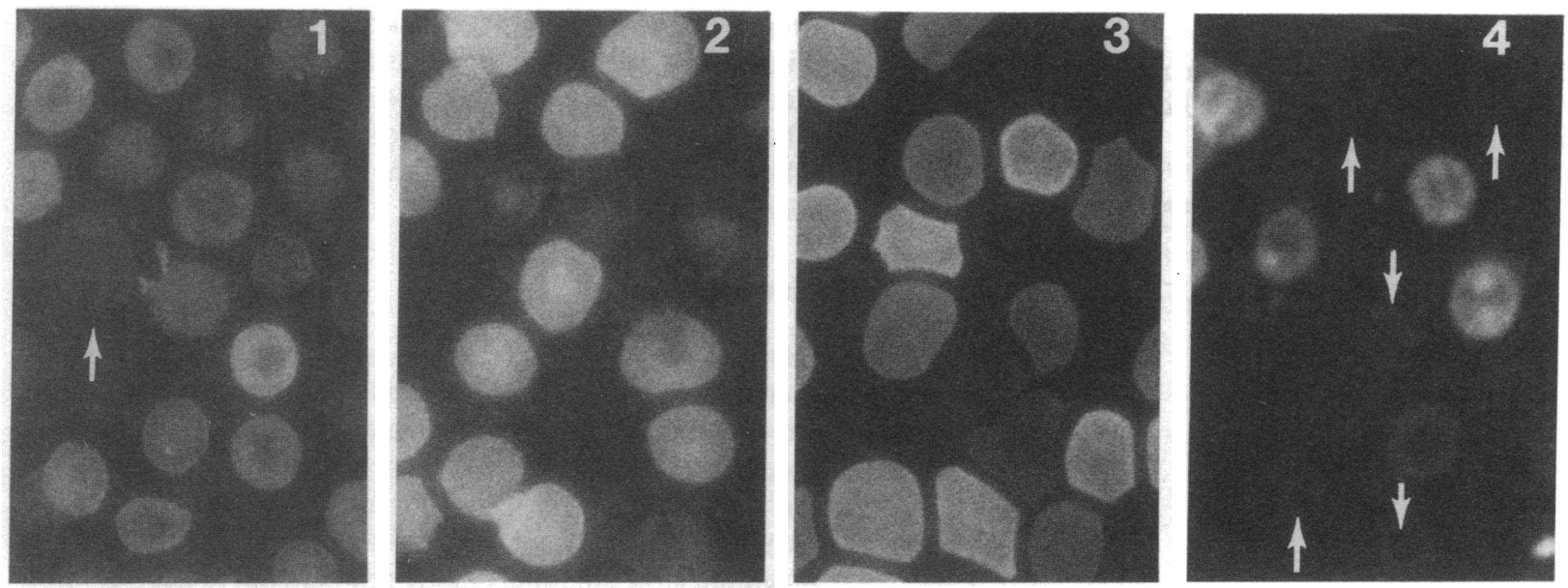

Figure 2. Photomicrographs of 15-lipoxygenase immunofluorescence in peripheral blood removed from a rabbit on day 15. Blood cells were separated into four fractions by buoyant density. Fraction 1 included immature reticulocytes (arrow), and many cells showed little fluorescence. Fractions 2 and 3 contained substantial numbers of reticulocytes of intermediate age which showed intense immunofluorescence. Fraction 4 contained many late reticulocytes with less intense staining and mature red blood with no staining (arrows). 

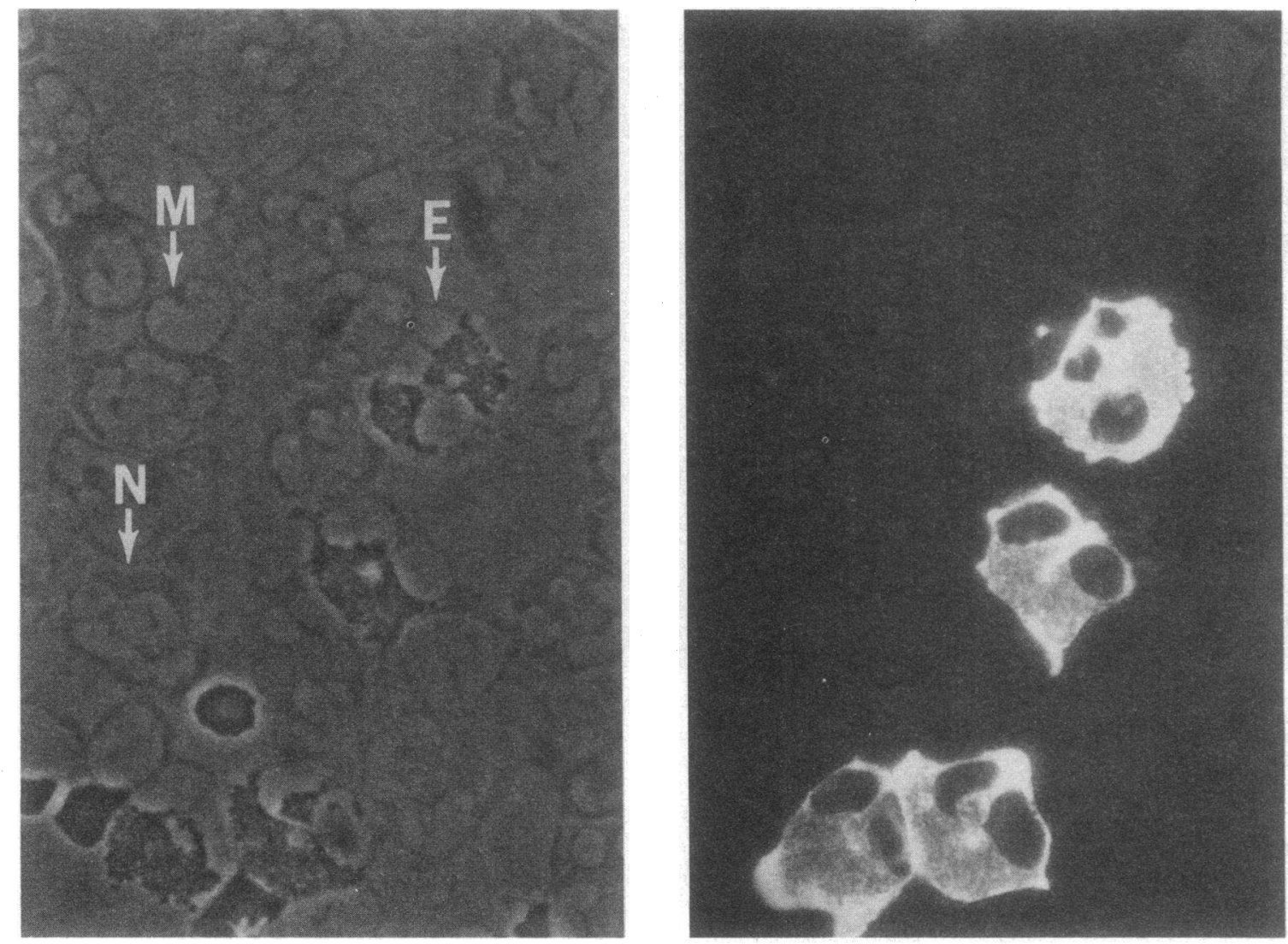

Figure 3. Photomicrographs of human peripheral blood leukocytes. Phase microscopy $(l e f t)$ differentiated mononuclear cells $(M)$, neutrophils $(N)$, and eosinophils $(E)$. Immunofluorescence (right) showed positive staining of eosinophils only.

the values for production of 15-LO were similar among subjects (Table I).

Only eosinophils showed 15-LO immunofluorescence. In each case, the fluorescence was localized to the cytoplasm and excluded the nucleus and the cytoplasmic granules. The immunofluorescence was quenched by recombinant $15-\mathrm{LO}$. In the two subjects who showed no 15-LO activity, no eosinophils were found in the blood smears, and no 15-LO immunofluorescence was present.

Table I. Relationship between the percentage of eosinophils (Eos), neutrophils (Neut), 15-lipoxygenase (LO) activity and immunofluorescence

\begin{tabular}{ccccccc}
\hline & \multicolumn{2}{c}{ Cell diff. (\%) } & & \multicolumn{2}{c}{ 15-HETE formatioon } & \\
\cline { 2 - 3 } Subject & Eos & Neut & & $\left(/ 10^{6}\right.$ cells) & $\left(/ 10^{6}\right.$ eos $)$ & $\begin{array}{c}\text { Fuorescence } \\
\text { (15-LO) }\end{array}$ \\
\hline 1 & $<1$ & $>99$ & & ND & - & None \\
2 & $<1$ & $>99$ & ND & - & None \\
3 & 21 & 79 & & 155 & 740 & Eos only \\
4 & 56 & 44 & 386 & 690 & Eos only \\
5 & 58 & 42 & 432 & 745 & Eos only \\
6 & 81 & 19 & 704 & 869 & Eos only \\
7 & 85 & 15 & 508 & 597 & Eos only \\
\hline
\end{tabular}

15-Lipoxygenase activity was evaluated by measuring 15-HETE formation (ng per $10^{6}$ leukocytes) formed from arachidonic acid (160 $\mu \mathrm{M})$. ND, not detected.
Human airways. We examined human airways by three methods: epithelial cells recovered from scrapings obtained postmortem, epithelial cells dissociated from postmortem tracheas, and whole sections of human airways (trachea and bronchi) obtained postmortem. In specimens of airway epithelium obtained from tracheas postmortem by scraping, both ciliated and basal cells were identified by phase microscopy (Fig. 4, upper panels). No goblet cells were found. Both ciliated (Fig. 4, lower panels) and basal cells showed fluorescent staining of the cytoplasm and exclusion of the nucleus, with marked variation in intensity of staining among cells. Fluorescence was inhibited selectively with the addition of $15-\mathrm{LO}$ antigen. Whole sections of human trachea and bronchi also showed 15-LO fluorescence in basal cells and ciliated cells of the epithelium, but connective tissue did not fluoresce.

\section{Discussion}

This study represents the first description of the immunocytochemical localization of 15-LO in blood and airway cells from any species. Antibodies to human 15-LO have only recently become available and are now shown to be capable of identifying 15-LO expression on a cellular level. Prior studies using enzymatic assays have been limited due to the difficulty in obtaining large amounts of pure cell material to analyze cellular localization. Whether high levels of expression by contaminating cells in such preparations are the source of enzyme activity has been a continual problem. For example, whereas it is 

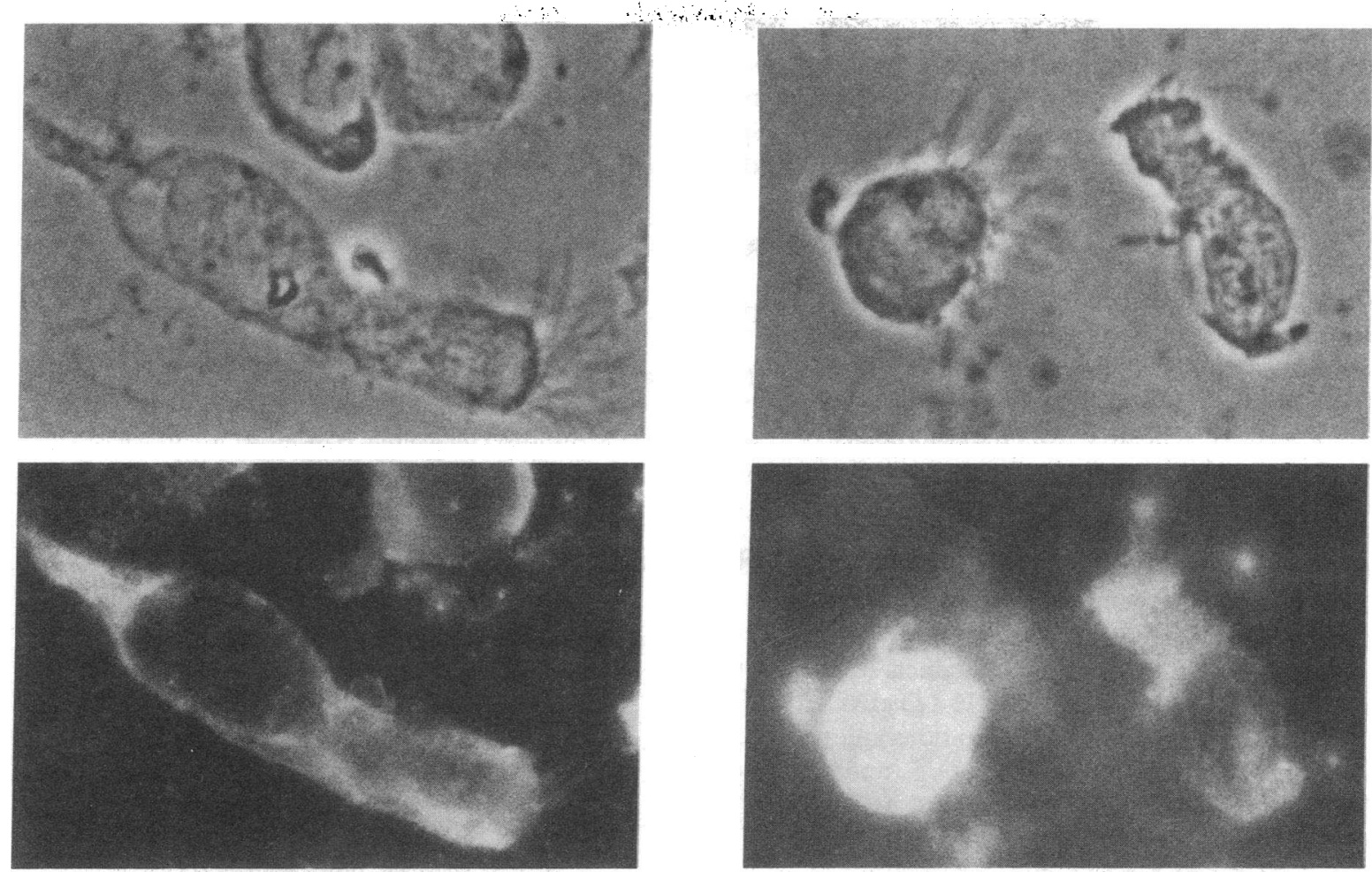

Figure 4. Photomicrographs of human airway epithelial cells isolated by scraping from a trachea postmortem. Phase microscopy (upper panels) identified ciliated cells. Immunofluorescence (lower panels) showed positive selective staining of the cytoplasm with marked variation in intensity of staining among cells.

clear that eosinophils have an active 15-LO (4), it has been much less clear whether other granulocytes, particularly neutrophils, express 15-LO. The current study presents evidence that 15-LO is a cytosolic enzyme which is highly tissue-specific and which is expressed variably from cell to cell in a given tissue. Among red blood cells, 15-LO is expressed at certain stages of reticulocyte differentiation. In leukocytes there is only expression in eosinophils. Within human airway, the expression of $15-\mathrm{LO}$ is localized to the airway epithelium, with variable expression among epithelial cells.

Investigators of the reticulocyte 15-LO have presented evidence from enzyme activity and immunoblots suggesting that the red blood cell enzyme is cell-specific $(2,3)$. Our study presents immunological evidence that the 15-LO of reticulocytes, eosinophils, and the airway epithelium are related. This is supported by immunoblots on crude preparations reported previously (17). Although the reason that prior studies concluded that the red blood cell enzyme is unique to that cell may be due in part to species differences, it is also possible that the screening assays used were not sensitive enough to detect low levels of specific tissue expression. Hence, assays on whole lung could potentially miss airway epithelial 15-LO expression and similarly, assays on leukocytes that are not enriched with eosinophils may not detect enzyme expression.

The 15-LO is believed to play a major role in the differentiation of the reticulocyte into a mature erythrocyte because the enzyme is selectively able to degrade, through lipid peroxidation, the mitochondria that that cell must rid itself of to become a specialized red cell $(2,9)$. We have observed a correlation of 15-LO fluorescence with reticulocyte differentiation showing that fluorescence was limited to developing red cells and was not detected in mature cells. Importantly, not all reticulocytes of all ages (as judged by buoyant density) expressed 15-LO. Large, young reticulocytes did not express 15-LO. A greater percentage of maturing reticulocytes exhibited fluorescence than did younger reticulocytes. In addition, 15-LO was diminished in late reticulocytes and was absent from mature red blood cells. These findings suggest that the enzyme is induced at some stage during the later phase of reticulocyte differentiation and is turned off completely at the stage of a mature red cell. This is entirely consistent with the studies on reticulocyte maturation performed in vivo $(2,9)$. Although the current study was not designed to monitor the appearance of 15-LO during the cellular events that occur during reticulocyte maturation, it is evident that in vitro and in vivo systems can be designed to do so and that the immunofluorescent detection of 15-LO should prove invaluable in correlating the role of the enzyme in these events.

Our results suggest that among leukocytes there is a preferential expression of 15-LO in eosinophils. In particular, there appears to be no evidence for neutrophil expression of 15-LO. Neutrophil fractions that contain eosinophils have activity that can be accounted for by the accompanying eosinophils, and the fluorescence in these fractions is localized to the eosinophils. This finding of preferential expression of 15-LO in eosinophils might appear to contrast with prior studies on leukocyte 15-LO in which the predominant cell type was the neutrophil $(5,22-$ 24). However, these prior studies did not report the percentage of contaminating eosinophils nor was the cell source among leukocytes a focus of these studies. The original studies on leu- 
kocyte $15-\mathrm{LO}$ by Turk et al. made the point that $15-\mathrm{LO}$ activity correlated with the percentage of eosinophils in mixed leukocyte preparations (4). The amount of 15-LO activity determined by these investigators and confirmed by later studies in our group (6) can readily explain the levels of 15-LO activity reported in mixed leukocyte preparations. For example, 15hydroxyicosatetraenoic acid (HETE) formation from eosinophils can be as high as $1,000 \mathrm{ng} / 10^{6}$ cells $(4,6,25)$. Therefore, a leukocyte preparation of $2 \times 10^{7}$ cells containing 4-8\% eosinophils, which is within normal range after mononuclear cells are removed, can produce up to $1,600 \mathrm{ng}$ of 15 -HETE. This amount of 15-HETE from the contaminating eosinophils can exceed the amount of 5-HETE generated from the accompanying, more abundant, neutrophils.

Conditions of maximal stimulation of 15-HETE involve cytotoxic doses of arachidonic acid, and this may account for the speculation that cell death activates $15-\mathrm{LO}(23)$. We considered the possibility that sonicated cell preparations of neutrophils might "unmask" 15-LO activity, but such preparations (>99\% neutrophils) failed to produce 15-LO products (data not shown). Furthermore, RNA blots using the 15-LO cDNA do not identify 15-LO mRNA in leukocyte preparations unless they are highly enriched for eosinophils (Conrad, D. J., and E. Sigal, unpublished observations). Therefore, we find no evidence for a neutrophil 15-LO. Nevertheless, we cannot rule out the possibility 15-LO expression can be induced in neutrophils as it is in red cells. The regulation of 15-LO expression, especially in disease states, is a current area of research.

There have been no prior studies of 15-LO localization performed on lung or airway. Although freshly isolated epithelial cells are known to exhibit 15-LO activity, the preferential localization of 15-LO to airway epithelium has not been previously established. Furthermore, the loss of activity that is seen during the culture of airway epithelial cells (Sigal, E., unpublished observations) has suggested that either 15-LO expression is downregulated during culture or the culture methods select out certain epithelial cells that do not express 15-LO. The current study clearly establishes the preferential localization of 15-LO to the airway epithelium and furthermore suggests that the enzyme resides in both basal and ciliated populations of epithelial cells. Culture conditions designed to modify the phenotype of cultured epithelial cells may result in active expression of 15LO. The immunofluorescent technique is ideally suited to explore this induction of 15-LO expression in that only a small number of cells are required to examine a large number of culture conditions.

Although 15-LO may respond to stimuli in the airway lumen and generate pro-inflammatory mediators as a protective mechanism, it is also possible that there is a separate, intracellular role for the enzyme. The cross-reactivity of the 15-LO in different cell types suggests a common biological role. Perhaps, in addition to generating inflammatory mediators in epithelial cells (and eosinophils), the 15-LO in these cells is involved in the type of membrane remodeling and degradation events seen in the differentiation of reticulocytes where causative action of 15-LO lipid peroxidation has been implicated.

A characteristic and consistent feature of this study was that 15-LO expression varied from cell to cell and was highly tissuespecific. This underscores the importance of examining expression on the cellular level and further suggests that other cell types in other tissues or in other pathological conditions may exhibit unrecognized expression of this enzyme. A case in point is the recent detection of 15-lipoxygenase in lipid-laiden, monocyte-derived macrophages of atherosclerotic lesions (26). Such studies are likely to reveal additional insight into the biological actions of 15-lipoxygenase.

\section{Acknowledgments}

The authors thank Patty Snell for manuscript preparation.

This study was supported in part by National Institutes of Health (NIH) Program Project Grant HL-24136. E. Sigal is a recipient of NIH Clinical Investigator Award HL-02047 and an Arthritis Investigator Award from the Arthritis Foundation. A. Schuster is the recipient of a fellowship from the Deutsche Forschungsgemeinschaft.

\section{References}

1. Murray, J. J., and A. R. Brash. 1988. Rabbit reticulocyte lipoxygenase catalyzes specific 12(S) and 15(S) oxygenation of arachidonoyl-phosphatidylcholine. Arch. Biochem. Biophys. 263:514-523.

2. Rapoport, S. M., T. Schewe, R. Wiesner, W. Halangk, P. Ludwig, M. Janicke-Hohne, C. Tannert, C. Hiebsch, and D. Klat. 1979. The lipoxygenase of reticulocytes. Purification, characterization and biological dynamics of the lipoxygenase: its identity with the respiratory inhibitors of the reticulocyte. Eur. $J$. Biochem. 96:545-561.

3. Kroschwald, P., A. Kroschwald, H. Kuhn, P. Ludwig, B. J. Thiele, M. Hohne, T. Schewe, and S. M. Rapoport. 1989. Occurrence of the erythroid cell specific arachidonate 15-lipoxygenase in human reticulocytes. Biochem. Biophys. Res. Commun. 160:954-960.

4. Turk, J., R. L. Mass, A. R. Brash, I. L. J. Roberts, and J. A. Oates. 1982. Arachidonic acid 15-lipoxygenase products from human eosinophils. J. Biol. Chem. 257:7068-7076.

5. Soberman, R. J, T. W. Harper, D. Betteridge, R. A. Lewis, and K. F. Austen. 1985. Characterization and separation of the arachidonic acid 5-lipoxygenase and linoleic acid $\omega-6$ lipoxygenase (arachidonic acid 15-lipoxygenase) of human polymorphonuclear leukocytes. J. Biol. Chem. 260:4508-4515.

6. Sigal, E., D. Grunberger, J. R. Cashman, C. S. Craik, G. H. Caughey, and J. A. Nadel. 1988. Arachidonate 15-lipoxygenase from human eosinophilenriched leukocytes: partial purification and properties. Biochem. Biophys. Res. Commun. 150:376-383.

7. Hamberg, M., P. Hedqvist, and K. Radegran. 1980. Identification of 15-hydroxy-5,8,11,13-eicosatetraenoic acid (15-HETE) as a major metabolite of arachidonic acid in human lung. Acta Physiol. Scand. 110:219-221.

8. Hunter, J. A., W. E. Finkbeiner, J. A. Nadel, E. J. Goetzl, and M. J. Holtzman. 1985. Predominant generation of 15-lipoxygenase metabolites of arachidonic acid by epithelial cells from human trachea. Proc. Natl. Acad. Sci. USA. 82:4633-4637.

9. Kuhn, H., and A. R. Brash. 1990. Occurrence of lipoxygenase products in membranes of rabbit reticulocytes. Evidence for a role of the reticulocyte lipoxygenase in the maturation of red cells. J. Biol. Chem. 265:1454-1458.

10. Shak, S., H. D. Perez, and I. M. Goldstein. 1983. A novel dioxygenation product of arachidonic acid possesses potent chemotactic activity for human polymorphonuclear leukocytes. J. Biol. Chem. 258:14948-14953.

11. Goetzl, E. J., M. J. Phillips, and W. M. Gold. 1983. Stimulus specificity of the generation of leukotrienes by dog mastocytoma cells. J. Exp. Med. 158:731737.

12. Marom, A., J. H. Shelhamer, F. Sun, and M. Kaliner. 1983. Human airway monohydroxyeicosatetraenoic acid generation and mucus release. J. Clin. Invest. 72:122-127.

13. Dahlen, S., L. Franzen, J. Raud, C. N. Serhan, P. Westlund, E. Wikstrom, T. Bjorck, H. Matsuda, S. E. Webber, C. A. Veale, T. Puustinen, J. Haeggstrom, K. C. Nicolaou, and B. Samuelsson. 1988. Actions of lipoxin $A_{4}$ and related compounds in smooth muscle preparations and on the microcirculation in vivo. In: Lipoxins: Chemistry Biosynthesis and Biological Activities. P.-Y. K. Wong and C. N. Serhan, editors. Plenum Publishing Corp., New York. 107-130.

14. Kirsch, C. M., E. Sigal, T. D. Djokic, P. D. Graf, and J. A. Nadel. 1988. An in vivo chemotaxis assay in the dog trachea: evidence for chemotactic activity of 8,15-diHETE. J. Appl. Physiol. 64:1792-1795.

15. Sigal, E., D. Grunberger, C. S. Craik, G. H. Caughey, and J. A. Nadel. 1988. Arachidonate 15-lipoxygenase ( $\omega-6$ lipoxygenase) from human leukocytes: purification and structural homology to other mammalian lipoxygenases. J. Biol. Chem. 263:5328-5332. 
16. Sigal, E., C. S. Craik, E. Highland, D. Grunberger, L. L. Costello, R. A. F. Dixon, and J. A. Nadel. 1988. Molecular cloning and primary structure of human 15-lipoxygenase. Biochem. Biophys. Res. Commun. 157:457-464.

17. Sigal, E., D. Grunberger, E. Highland, C. Gross, R. A. F. Dixon, and C. S. Craik. 1990. Expression of cloned human reticulocyte 15-lipoxygenase and immunological evidence that 15 -lipoxygenases of different cell types are related. $J$. Biol. Chem. 265:5113-5120.

18. Rapoport, S., J. Schmidt, and S. Prehn. 1985. Maturation of rabbit reticulocytes: susceptibility of mitochondria to ATP-dependent proteolysis is determined by the maturational state of reticulocyte. FEBS (Fed. Eur. Biochem. Soc.) Lett. 183:370-373.

19. Yazdanbakhsh, M., C. M. Eckmann, M. D. Boer, and D. Roos. 1987 Purification of eosinophils from normal blood, preparation of eosinoplasts and characterization of their functional response to various stimuli. Immunology. 60:123-129.

20. Widdicombe, J. H., C. B. Basbaum, and E. Highland. 1981. Ion contents and other properties of isolated cells from dog tracheal epithelium. Am. J. Physiol. 241:C184-C192.

21. Filley, W. V., S. J. Ackerman, and G. J. Gleich. 1981. An immunofluores- cent method for specific staining of eosinophil granule major basic protein. $J$. Immunol. Methods. 47:227-238.

22. Fruteau de Laclos, B. P. Braquet, and P. Borgeat. 1984. Characteristics of leukotriene (LT) and hydroxy eicosatetranoic acid (HETE) synthesis in human leukocytes in vitro: effect of arachidonic acid concentration. Prostaglandins Leukotrienes and Medicine. 13:47-52.

23. McGuire, J., J. McGee, N. Crittenden, and F. Fitzpatrick. 1985. Cell damage unmasks 15-lipoxygenase activity in human neutrophils. J. Biol. Chem. 260:8316-8319.

24. Nichols, R. C., and J. Y. Vanderhoek. 1990. 5-Hydroxyeicosanoids selectively stimulate the human neutrophil 15 -lipoxygenase to use endogenous substrate. J. Exp. Med. 171:367-375.

25. Holtzman, M. J., A. Pentland, N. L. Baenziger, and J. R. Hansbrough. 1989. Heterogeneity of cellular expression of arachidonate 15-lipoxygenase: implications for biological activity. Biochim. Biophys. Acta. 1003:204-208.

26. Ylä-Herttuala, S., M. E. Rosenfeld, S. Parthasarathy, C. K. Glass, E. Sigal, J. L. Witztum, and D. Steinberg. 1990. Colocalization of 15-lipoxygenase mRNA and protein with epitopes of oxidized low density lipoprotein in macrophage-rich areas of atherosclerotic lesions. Proc. Natl. Acad. Sci. USA. 87:6959-6963. 